\title{
Is chlorhexidine mouth rinse, used as a mono-therapy or adjunct with oral hygiene, effective at reducing plaque growth and gingival inflammation?
}

\author{
Authors: \\ Andre W. van Zyl ${ }^{1}$ \\ Johan Hartshorne 2 \\ Alonso Carrasco-Labra ${ }^{3,4}$ \\ Affiliations: \\ ${ }^{1}$ Department of Periodontics \\ and Oral Medicine, University \\ of Pretoria, South Africa \\ ${ }^{2}$ Visiting Professor: \\ Department of Periodontics \\ and Oral Medicine, School \\ of Dentistry, University of \\ Pretoria, Private Practice, \\ Tyger Valley, South Africa \\ ${ }^{3}$ Department of Oral and \\ Maxillofacial Surgery and \\ Evidence-Based Dentistry \\ Unit, Universidad de \\ Chile, Chile \\ ${ }^{4}$ Department of Clinical \\ Epidemiology and \\ Biostatistics, McMaster \\ University, Canada \\ Correspondence to: \\ Johan Hartshorne \\ Email: \\ jhartshorne@kanonberg.co.za \\ Postal address: \\ PO Box 6223, Welgemoed \\ 7538 , South Africa \\ How to cite this article: \\ Van Zyl A, Hartshorne \\ J, Carrasco-Labra A. Is \\ chlorhexidine mouth rinse, \\ used as a mono-therapy or \\ adjunct with oral hygiene, \\ effective at reducing \\ plaque growth and gingival \\ inflammation? Open J \\ Implant Dent. 2013;1(1), Art. \\ \#5, 3 pages. http://dx.doi. \\ org/10.4102/ojid.v1i1.5

\section{Copyright:} \\ (C) 2013. The Authors. \\ Licensee: AOSIS \\ OpenJournals. This work \\ is licensed under the \\ Creative Commons \\ Attribution License. \\ Read online:

This study describes a systematic review of the best available evidence on the effectiveness of a chlorhexidine mouth rinse as a mono-therapy or as an adjunct to mechanical oral hygiene against plaque growth and gingival inflammation. Medline, EMBASE and the Cochrane Central register of Controlled Trials were searched up to April 2011. Randomised controlled clinical trials that compared chlorhexidine to placebo or controlled mouth rinses or regular oral hygiene for a minimum duration of at least four weeks amongst gingivitis patients ( $\geq 18$ years of age) were included. A total of 30 publications fulfilled the selection criteria. Clinical parameters measured at baseline and end of trial were plaque, gingival inflammation, bleeding and staining. Chlorhexidine reduced plaque by $33 \%$ and gingivitis by $26 \%$ compared to a placebo or a control mouth rinse. The investigators concluded that when used together with oral hygiene, chlorhexidine mouth rinses provide significant reductions in plaque and gingivitis scores in gingivitis patients, but a significant increase in staining compared to placebo or control mouth rinses.

\section{Focus article}

Van Strydonck DAC, Slot DE, Van der Velden U, Van der Weijden F. Effect of a chlorhexidine mouth rinse on plaque, gingival inflammation and staining in gingivitis patients: a systematic review. J Clin Periodontol. 2012;39:1042-1055.

\section{Background}

Dental plaque control and elimination of gingival infection and inflammation are important to improve and maintain oral health. Chlorhexidine gluconate ( $\mathrm{CHX})$ is commonly used as the active ingredient in oral rinses to control microbial plaque and gingivitis. $\mathrm{CHX}$ is a cationic bisbiguanide biocide with broad spectrum antibacterial activity. ${ }^{1}$ Most bacteria and surface structures in the oral cavity, including the surfaces of teeth and mucous membranes, are negatively charged (anionic), and will therefore bind strongly to the positively charged CHX.

$\mathrm{CHX}$ is retained in the oral cavity for at least 12 hours after rinsing and released slowly into the oral fluids. ${ }^{2}$ Its antimicrobial effect persists because it binds strongly to proteins in the mucosa. $\mathrm{CHX}$ has both rapid bactericidal and prolonged bacteriostatic mechanisms of action against a wide range of Gram-positive and Gram-negative bacteria. Its primary mechanism of action is through bacterial cell membrane disruption.

$\mathrm{CHX}$ at concentrations below $0.2 \%$ is generally considered safe and has an inherent advantage over antibiotics because it does not produce resistant microorganisms, or cause overgrowth of potentially opportunistic organisms or other adverse changes in the oral microbial ecosystem during prolonged use. ${ }^{3}$

Multiple comparative clinical studies have been performed to demonstrate the efficacy of different $\mathrm{CHX}$ mouth rinse formulations against plaque growth, gingivitis and staining. However, no systematic evaluation of the effectiveness of $\mathrm{CHX}$ mouth rinses has yet been performed.

\section{Appraisal of study methodology and validity of the results} This review addressed the following clinical question:

Does CHX mouth rinse as a mono-therapy or as an adjunct to mechanical oral hygiene in randomised controlled clinical trials (RCTs) have an effect on plaque and parameters of gingival inflammation as compared to a placebo rinse, a control rinse or regular oral hygiene when used in healthy adults with gingivitis over a minimum of four weeks? 
Customised search strategies based on the focus question and appropriate eligibility criteria were developed for MEDLINE-PubMed, EMBASE and the Cochrane Central Register of Controlled Trials databases. All databases were searched up to April 2011. The search for relevant studies was detailed and exhaustive. A total of 30 publications fulfilled the required selection and eligibility criteria for inclusion in the review.

Two reviewers scored the methodological quality of the included studies and assessed the risk of bias. The potential risk of bias was low in 9 of the 30 primary studies, moderate for 10 studies and high for 11 studies. Allocation concealment was reported in only 7 of the studies. Most primary studies complied with the following criteria:

- random allocation

- balanced experimental groups

- blinded to the examiner and patient

- appropriate comparison condition

- reporting of loss to follow up.

The selection and assessment of studies can be considered adequate and reproducible. Two studies did not define their eligibility criteria and two had missing or insufficient information. Industry funding was reported for 15 of the studies.

This review reasonably satisfied all the internal, external and statistical validity assessment criteria. Overall, we concluded that the methods of the systematic review and the methodological quality of the primary studies were moderate to strong.

\section{Results}

The reviewed studies varied with regard to study design, evaluation period, population demographics and the rinsing regimen. All but one study used a cross-over instead of a parallel study design. The ages of the participants ranged from 17 to 66 years. The concentrations of $\mathrm{CHX}$ oral rinses varied between $0.05 \%$ and $0.2 \%$; however, most studies used a concentration of $0.12 \%$. The $\mathrm{CHX}$ formulation contained alcohol in all the studies. The majority of subjects rinsed twice daily and the rinsing volume varied between $10 \mathrm{~mL}$ and 15 $\mathrm{mL}$. Unsupervised rinsing time varied between 30 seconds and 60 seconds. No baseline prophylaxis was conducted in 11 of the studies. The number of participants who smoked was reported in 6 of the studies. The effect of smoking on the outcome variables was not analysed. Smokers were excluded from 3 of the studies. A meta-analysis of 13 of the included studies was performed to compare the effects of a $\mathrm{CHX}$ mouth rinse as an adjunct to oral hygiene on plaque, gingivitis, bleeding and staining to a placebo rinse, a control rinse or regular oral hygiene.

Point estimates of mean differences between base and endof-trial scores for papillary bleeding $\left(\chi^{2}=0.08 ; p=0.77 ; I^{2}=\right.$ $0 \%)$ and bleeding on marginal probing $\left(\chi^{2}=1.21 ; p=0.27\right.$;
$I^{2}=18 \%$ ) appeared to be similar and consistently favoured $\mathrm{CHX}$. The end scores for plaque, gingival index and staining showed moderate to considerable inconsistency. Reasonable overlap of confidence intervals between studies was observed for all the outcome variables, indicating that there was no significant difference in the results of the individual studies.

Although there was significant variability between individual studies, no statistically significant differences were observed between subgroups for plaque scores $\left(p=0.60 ; I^{2}=0 \%\right)$, papillary bleeding $\left(p=0.77 ; I^{2}=0 \%\right)$, gingival scores $\left(p=0.49 ; l^{2}=0 \%\right)$ or bleeding on marginal probing $(p=0.27$; $\left.I^{2}=0 \%\right)$. Statistically significant differences were, however, observed between subgroups for staining scores, which could probably be ascribed to differences in rinsing regimens and concentration of rinses used by participants.

The meta-analysis showed significant weighted mean differences for all the treatment outcome parameters, favouring CHX. Overall, the evidence demonstrated that a CHX mouth rinse is effective in controlling plaque and gingivitis. The investigators also concluded that the effect of $\mathrm{CHX}$ as an adjunct was consistent throughout the individual studies. CHX reduced plaque by $33 \%$ and gingivitis by $26 \%$ relative to the controls.

Significantly more staining was demonstrated amongst CHXrinsing groups. Increased calculus formation and altered taste sensation were also frequently observed. Burning sensations, mucosal lesions and hypersensitivity were less frequently observed as adverse events.

\section{Applicability of the results}

Participants in the RCTs assessed in this review are quite similar to the ones treated in everyday general practice. The clinical effectiveness and safety of CHX oral rinses have not been established in children under the age of 18. Caution should also be exercised when $\mathrm{CHX}$ is administered to nursing mothers because it is not known whether this drug is secreted in milk.

Using CHX prior to surgical procedures and as an adjunct to non-surgical management of periodontal disease to reduce bacterial load should not be underestimated. However, clinicians should take note that anionic compounds such as surfactants, thickeners and emulsifiers that are commonly used in toothpastes deactivate CHX and instruct their patients accordingly. Therefore, CHX mouth rinses should be used at least 30 minutes after brushing teeth. For optimal effectiveness, it is also recommended that eating, drinking and smoking should be avoided for at least an hour after rinsing.

\section{Clinical resolution}

This study presented strong evidence that the adjunctive use of CHX mouth rinse as part of regular oral hygiene has 
anti-plaque and anti-gingivitis effects in gingivitis patients. $\mathrm{CHX}$ is a simple, inexpensive and effective adjunct to reduce bacterial load, thus yielding significant benefits with regard to patients' oral health.

\section{Acknowledgements}

AOSIS is thanked for contributing towards the copyediting and publishing costs of this article.

\section{Competing interests}

The authors declare that they have no financial or personal relationship(s) that may have inappropriately influenced them in writing this article.

\section{Authors' contributions}

A.v.Z. and J.H. contributed towards clinical content and synthesis. A.C-L. and J.H. contributed towards research method and evaluation of the quality of the presented evidence.

\section{References}

1. Hugo WB, Longworth AR. The effect of chlorhexidine on the electrophoretic mobility cytoplasmic constituents, dehydrogenase activity and cell walls of Escherichia col and Staphylococcus aureus. J Pharm Pharmcol. 1996;18(9):569-578. http://dx.doi. org/10.1111/j.2042-7158.1966.tb07935.x

2. Addy $M$, Jenkins $S$, Newcombe R. The effect of some chlorhexidine-containing mouthrinses on salivary bacterial counts. J Clin Periodontol. 1991;18:90-93. http: /dx.doi.org/10.1111/j.1600-051X.1991.tb01694.x, PMid:2005231

3. Kolahi J, Soolari A. Rinsing with chlorhexidine gluconate solution after brushing and flossing teeth: a systematic review of effectiveness. Quintessence Int. 2006;37:605-612. PMid:16922019 